\title{
Delay Performance of Packet Scheduling Algorithms in LTE_based Machine-to- Machine Communications
}

\author{
A.I.A. Jabbar, $\mathrm{PhD}$ \\ Engineering College \\ Department of Electrical Engineering \\ Mosul University
}

\author{
Fawaz Y. Abdullah \\ Engineering College \\ Department of Electrical Engineering \\ Mosul University
}

\begin{abstract}
Introducing efficient M2M networks into the LTE /LTE-A networks can be achieved with careful selection of vital parameter, such as the traffic load (due to a large number of $\mathrm{M} 2 \mathrm{M}$ devices) and the behavior of M2M traffic, which differs from traditional mobile traffic. In future, applications like health monitoring vital signs (ECG, heartbeat) and Remote diagnostics are considered to be, the primary M2M application areas.

This paper deals with various scheduling algorithms for resource allocation and their impact on real-time traffic in LTE networks. It investigates several traffic M2M models in healthcare monitoring system. It also evaluates the performance of M2M traffic in health monitoring with file transfer (FTP) and Voice users. The maximum delay (CQ) differences between the algorithms are obtained for medical applications. The simulation results indicate that the ehealthcare related data traffic has a significant influence on other LTE traffic.
\end{abstract}

\section{General Terms}

Computer Network, Wireless Networks, M2M,SchedulingAlgorithms.

\section{Keywords}

M2M, LTE networks, Traffic modeling, LTE data traffic, scheduling algorithms, Qos.

\section{INTRODUCTION}

M2M communications, or machine-type communications (MTC), is defined as a data communications among machines through the necessary infrastructures without the need for direct human management or control. In LTE, the high performance of the radio network can be realized with proper scheduling of resources especially, for real-time traffic like VoIP. The choice of proper scheduling strategies plays an important role in guaranteeing minimal E2E delay performance for both $\mathrm{M} 2 \mathrm{M}$ and traditional mobile services [1].

The 2011 ITU-T (International Telecommunication UnionTelecommunications) Technology Watch report notes that trends in e-Healthcare point towards increasing focus on personalized medicine standardized electronic health records and diagnostics [2,3]. Therefore, e-Healthcare is a key application of M2M currently under active consideration in different standards for such as ETSI TC e-Health, ETSI TC M2M, ITU-T Focus Group on M2M, etc. According to [4], M2M applications for e-Health enable the remote monitoring of patient health and fitness information. ETSI M2M defines $[5,6,7]$ some e-Health applications: remote disease management, aging independently, and personal fitness and health improvement $[8,10]$. Compared to the medical applications in support of illness management and aging alone, the support of personal fitness and health improvements will most likely require less frequent uploading of data and is more tolerant to delays [9].

From the application point of view, e-healthcare technologies for example usually apply indoor scenarios for patient monitoring. The authors in [12] explained the security issues of e-healthcare over mobile networks, and have proposed architecture to facilitate doctors and patients' communication within the hospital. The research of packet scheduling algorithms in the wireless channel have been studied for a long time [14, 15, and 16]. In reference [14], a comparative study of PQ, DWRR, and WFQ algorithms have been reviewed. A comparison for VoIP traffic based on FIFO, CQ, MWRR, and PQ algorithms is shown in reference [15]. In addition, in reference [16], simulation-based comparative analysis of FIFO, PQ, WFQ, and DWRR mechanism have been considered and in real-time application parameter, PQ is the minimum value rather than the other algorithms.

This paper, investigates the performance of several traffic M2M models in healthcare monitoring systems with different packet scheduling algorithms, it is organized as follows. Section two, presents and overview of M2M Communication in Healthcare. Section three, presents of packet scheduling algorithms .The simulation results presented and analyzed in Sections 4 and 5. Section 6 concludes the paper.

\section{M2M COMMUNICATION IN HEALTHCARE}

Fig.1illustrates the architecture of a wireless M2M system for e-Health applications. The ETSI framework consists of five key elements as described below [17]:

1) The M2M devices (MD), which are devices capable of transmitting data autonomously.

2) The M2M Area Network, also known as the capillary network, which is the short-range network interconnecting the MD and providing a link to the M2M gateway.

3) The M2M Gateway, which acts as a proxy between the MD and the network domain.

4) The M2M Access Communication Network, which connects the M2M gateway to the NETWORK, eventually reaching the M2M application server. The communication network can be broken down to the access network and the core network [18]. Conventional wireless technologies employed by the access network include Long Term Evolution (LTE), WiMAX and IEEE 802.11 WLAN.

5) The M2M Application Server, which is the middleware layer that provides data to the specific business applications. 
LTE-Advanced, is the wireless network segment considered in this paper. The MD is connect to LTE-Advanced via a conventional base station (eNodeB B). The eNodeB is in turn are connected to the Mobility Management Entity (MME), Serving Gateway (SGW) and Packet data network Gateway (P-GW). The M2M server not only acts a data sink for storing all sensory data from the M2M terminals but also provides these real-time data to a variety of $\mathrm{M} 2 \mathrm{M}$ applications for remote monitoring and management [17].

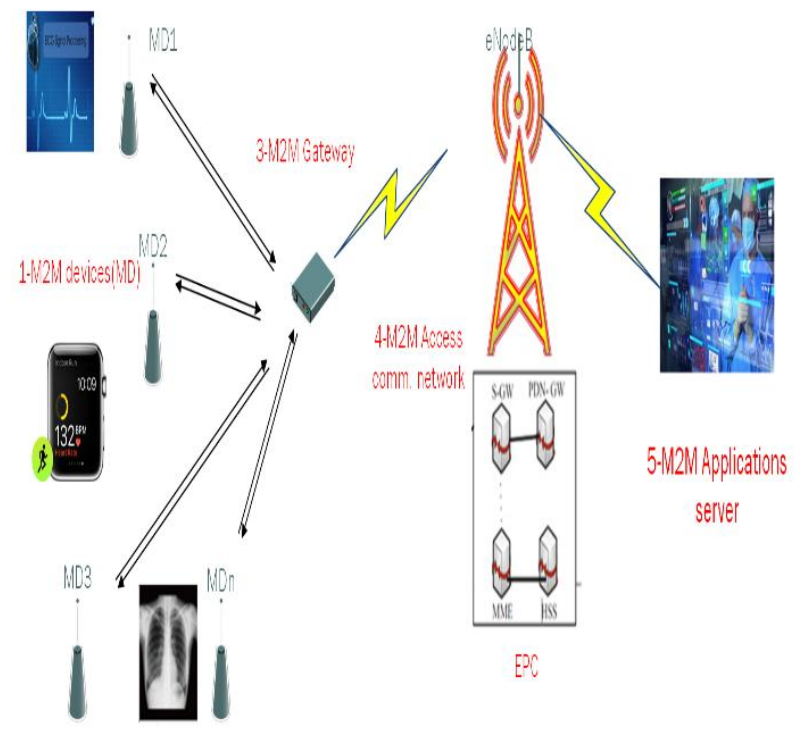

$$
\begin{aligned}
& \text { 2-M2M Area Network } \\
& \text { Fig. 1.Simplified (M2M) architecture fork domain } \\
& \text { connectivity for healthcare. }
\end{aligned}
$$

\subsection{M2M Traffic Models}

Several M2M traffic models for various e-healthcare M2M applications are listed in Table 1 have been investigated in this work. The primary parameters for M2M traffic model are the message size transmitted by the M2M device and intersend time i.e. time between transmissions of two consecutive M2M messages. The message size varies according to the M2M applications [13].

In M2M narrowband applications such as E-healthcare and intelligent traffic sensors, devices transmit small sized data. For instance, devices which are deployed for Electrocardiography (ECG) monitoring system send 1200 bytes per $1 \mathrm{~s}$. Whereas, devices which are deployed in broadband applications such as Picture Archiving and Communication System (PACS is the system that has replaced x-ray film with digital images [19] ) transmit large size data (100 Kbyte). PACS resolves many of the problems that were associated with the film. The film could only be available in one place at a time and would frequently result in delayed patient care if it were not immediately available to the referring physician. With PACS, patient studies can be viewed from any computer at any of our facilities or from a referring physician's office [19].
Table 1. List of e-healthcare M2M traffic models.

\begin{tabular}{|c|c|c|}
\hline M2M applications & $\begin{array}{c}\text { Message size } \\
\text { (bytes) }\end{array}$ & $\begin{array}{c}\text { Inter- } \\
\text { send } \\
\text { time } \\
\text { (s) }\end{array}$ \\
\hline $\begin{array}{l}\text { e-health monitoring vital } \\
\text { signs (heartbeat, blood } \\
\text { pressure, (SpO2), glucose } \\
\text { level, etc.) }\end{array}$ & 97 & 1 \\
\hline $\begin{array}{c}\text { Electrocardiography (ECG) } \\
\text { monitoring system }\end{array}$ & 1200 & 1 \\
\hline $\begin{array}{c}\text { Picture Archiving and } \\
\text { Communication System } \\
\text { (PAC).(high-resolution } \\
\text { medical images) }\end{array}$ & 100000 & 12 \\
\hline
\end{tabular}

\section{PACKET SCHEDULING ALGORITHMS}

Packet scheduling mechanism is one of the important items for QoS control [11]. Packet scheduling specifies the service policy of a queue within a node (e.g. an IP router, an EPC).One of the most important objectives of a scheduling scheme is to satisfy the QoS requirements of its users while efficiently utilizing the available bandwidth [21].

The performance of main packet scheduling algorithms such as-

- $\quad$ Priority Queuing (PQ).

- Custom Queuing (CQ).

- Weighted Fair Queuing (WFQ).

- Modified Weighted Round Robin (MWRR).

- $\quad$ First in First out (FIFO).

In LTE mobile networks has been studied to tuning the scheduling decisions, that is, decide which is the minimum programming period for an $\mathrm{M} 2 \mathrm{M}$ cluster to meet the probabilistic QoS targets and estimating the minimum LTE bandwidth reserved for M2M to support different M2M loads with prescribed QoS requirements.

\subsection{QoS Requirements for e-Health \\ Services.}

Typically-Health applications usually transfer highresolution medical images (PAC) and clinical/administrative data. Such applications generate traffic with very diverse network requirements, differing in required bandwidth, realtime versus non-real-time interactivity, and tolerance for packet loss. Often, an e-Health service will involve the simultaneous transmission of multiple media flows, such as the mobile emergency system including audio/video, medical images, and ECG signals.

\section{MODELING AND SIMULATION}

The model simulation is achieved according to the following specifications:-

- The LTE model consists of several nodes with LTE functionalities and protocols implemented as shown in Fig.2. In the simulation network topology, there are two cells represented by e-NodB 1 and e-NodB 2 connected to 
the EPC. The distance between eNode-Bs is around 6 $\mathrm{km}$.

- The suburban area is employed using the path loss model for all simulations.

- The simulation parameter settings given in Table 2. The LTE traffic for simulations includes voice, FTP and ehealthcare traffic as shown in Table 3 .

- The various e-healthcare traffic load performance scenarios are compared by analyzing the QoS performance of mobile devices with regular LTE uplink traffic.

- Two types of LTE uplink traffic (voice and file transfer) and three e-healthcare traffic are assumed.

- The number of voice is 20 (10 in each cell) sources and file transfer sources is 10 (5 in each cell) are deployed in all the scenarios. The voice users are mapped to the Guaranteed Bit Rate (GBR) bearer (priority 2), and the FTP users are assigned to the NonGBR bearer (priority 9). In the first scenario, 100 health devices (Message size $=97$ Bytes) are deployed in the cell. The 100 ECG devices with Message size $=1200$ Bytes in second scenario and 100PACS devices (Message size $=100$ Kbytes) in the third scenario. The e-healthcare users are mapped to the Guaranteed Bit Rate (GBR) bearer (priority 2).

- The different packet scheduling algorithms (FIFO, PQ, CQ, WFQ, and DWRR) implemented at the nodes EPC, Edge Router and E-Node B 1 \& E-Node B 2 for each scenario.

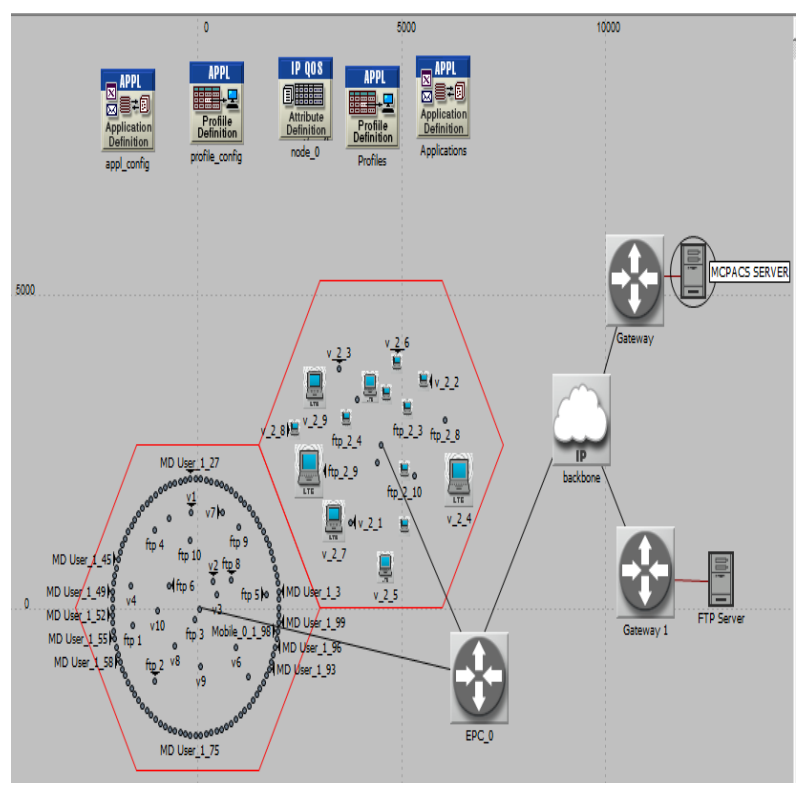

Fig. 2 OPNET project editor

Table 2. Simulation Parameters

\begin{tabular}{|c|c|}
\hline Parameter & Value \\
\hline 1-eNode-B & 2 cells \\
\hline System Model & 9 (QPSK) \\
\hline MCS & \\
\hline Channel Bandwidth & $20 \mathrm{MHz}$ \\
\hline
\end{tabular}

\begin{tabular}{|c|c|}
\hline & \\
\hline Path Loss Model & the suburban macrocell \\
\hline No. Tx Antenna & 3 \\
\hline No. Rx Antenna & $\mathbf{3}$ \\
\hline Antenna Gain & $15 \mathrm{dBi}$ \\
\hline Max. Transmission Power & $\mathbf{2 0 ~ W}$ \\
\hline SIMULATION TIME & $\mathbf{6 0 0}$ sec \\
\hline
\end{tabular}

Table 3. LTE traffic models

\begin{tabular}{|c|c|}
\hline Parameter & Value \\
\hline Voice user & $\begin{array}{c}\text { (10user for each cell) 2cells } \\
\text { are talkers }\end{array}$ \\
\hline Antenna Gain & $-1 \mathrm{dBi}$ \\
\hline Channel Model & suburban \\
\hline $\begin{array}{l}\text { Max. Transmission } \\
\text { Power }\end{array}$ & $0.5 \mathrm{~W}$ \\
\hline No. of $\mathrm{Tx} / \mathrm{Rx}$ Antennas & 1 \\
\hline Voice G.711 & (64 kbps) \\
\hline FTP Traffic Model & $\begin{array}{c}10 \text { Users(5 users for each } \\
\text { cell }\end{array}$ \\
\hline File size & 1 M bytes \\
\hline $\begin{array}{l}\text { File inter-request time } \\
\text { Uniform distribution }\end{array}$ & $(80-100 \mathrm{~s})$ \\
\hline MD e-healthcare user & $\begin{array}{c}\text { (100 M2M user for cell } \\
\text { number1) }\end{array}$ \\
\hline Channel Model & suburban \\
\hline $\begin{array}{l}\text { Max. Transmission } \\
\text { Power }\end{array}$ & $2 \mathrm{~W}$ \\
\hline No. of $T x / R x$ Antennas & 1 \\
\hline Antenna Gain & $14 \mathrm{dBi}$ \\
\hline $\begin{array}{l}\text { e-health traffic model } \\
\text { Frame size }\end{array}$ & 97 Bytes \\
\hline Frame inter-arrival time & $1 \mathrm{sec}$ \\
\hline $\begin{array}{l}\text { ECG traffic model Frame } \\
\text { size }\end{array}$ & 1200 Bytes \\
\hline Frame inter-arrival time & $1 \mathrm{sec}$ \\
\hline $\begin{array}{l}\text { PAC traffic model Frame } \\
\text { size }\end{array}$ & 100k Bytes \\
\hline Frame inter-arrival time & $10 \mathrm{sec}$ \\
\hline
\end{tabular}

\section{RESULTS AND ANALYSIS}

The delay performance of three e-healthcare applications in the systems with different Queueing algorithms is investigated in this section. The traffc models that are defined in Table 3 
are adopted. Fig. 3 shows the average M2M packet end-toend delay, by increasing the message size the average M2M packet end-to-end delay users' increases.

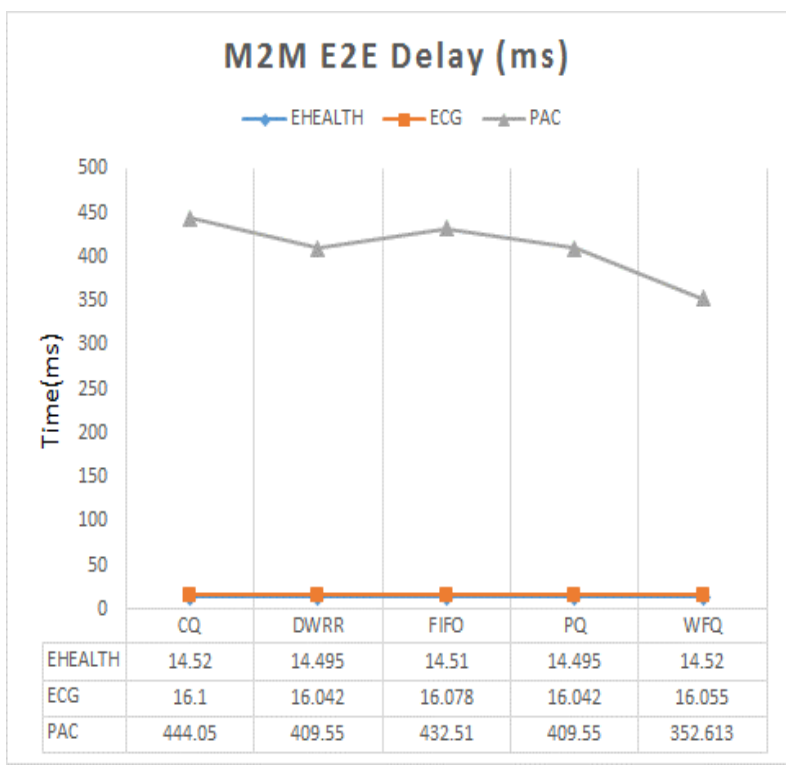

Fig.3.M2M packet end-to-end delay

The delay is small for DWRR and PQ algorithms compared to CQ, FIFO, and WFQ as shown in Fig.4. The maximum delay as (CQ), is observed between the algorithms for medical applications.

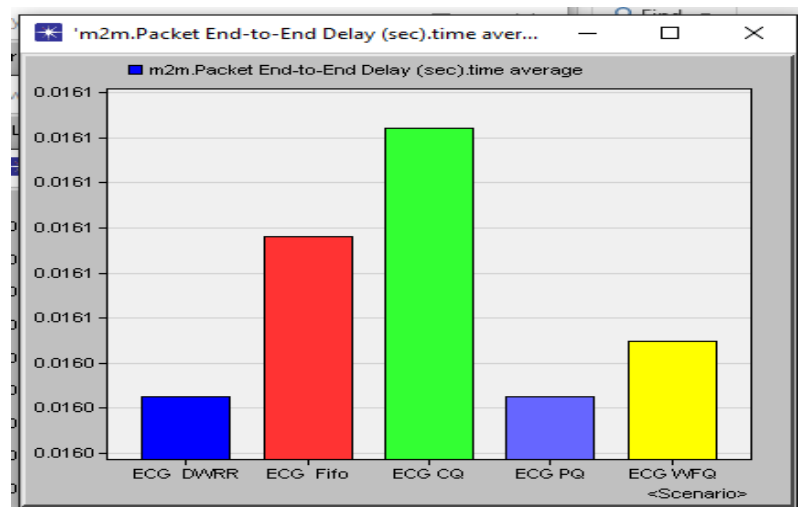

Fig.4. ECG packet end-to-end delay

Fig.5 depicts the performance of FTP traffic regarding file average upload time. The results in all the three e-healthcare traffic models clearly reflect the considerable amount of delay in file transfer users. By increasing the message size the average, upload time for FTP users increases. Fig. 6 depict the performance of voice users regarding voice packet E2E delay. All the three models show no significant impact of ehealthcare devices on LTE voice traffic (Table 4). The end-toend delay for voice packet is small for PQ algorithms compared to WFQ and FIFO as shown in Fig.6.

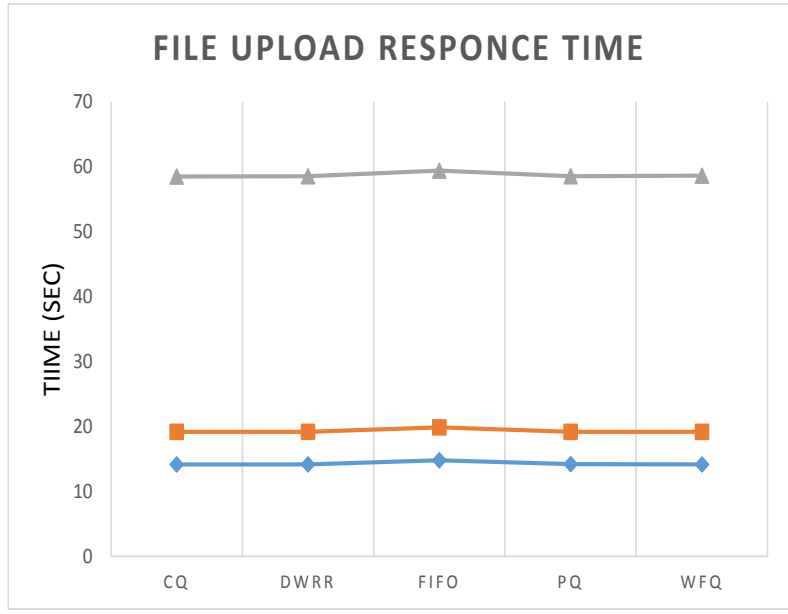

Fig.5. Average file uploads time.

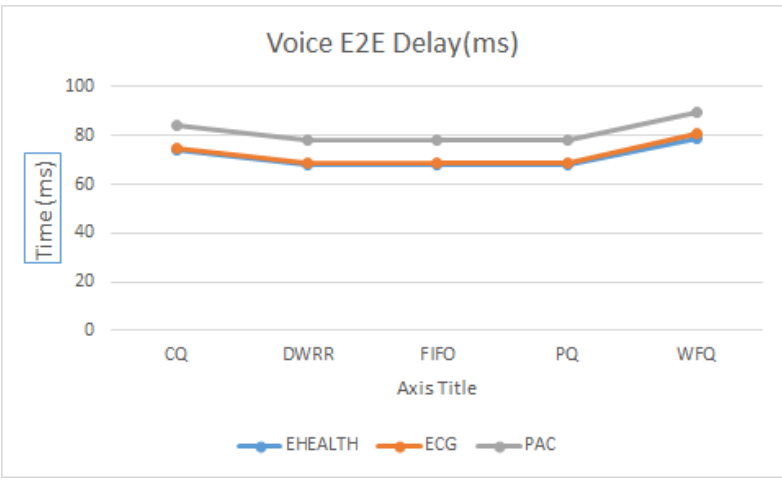

Fig.6.Voice packet end-to-end delay.

The average end-to-end (E2E) delay for the Voice traffic (in FIFO) is found $89.85 \mathrm{~ms}$. This value fulfills the ITU-R and the 3GPP standard requirements with up to $150 \mathrm{~ms}$ for one way Voice E2E delay to experience high quality. In fact, an E2E delay up to $250 \mathrm{~ms}$ is still quite satisfactory for the majority of users if we considered about $100 \mathrm{~ms}$ required the delay for packet processing and propagation delay in the LTE core network [22].

The UL/DL delay for different message size is illustrated in Fig. 7 and Fig. 8 respectively. The relation between the UL/DL delay and the message size is directly proportional. The results show that there is up to 0.08 seconds DL delay and up to 0.03 seconds UL delay. These results meet the requirements mentioned before, for the one-way voice radio UL/DL delay. The Packet Delay in the DL direction is less than the UL direction, although the resulting delay in both sides follows the same standard requirements of $100 \mathrm{~ms}$ oneway delay [22]. This is due to the higher power on the DL side from the eNBs which is around (20 watt), compared to the UE's power which is about (2 watt) in the UL side [23]. 


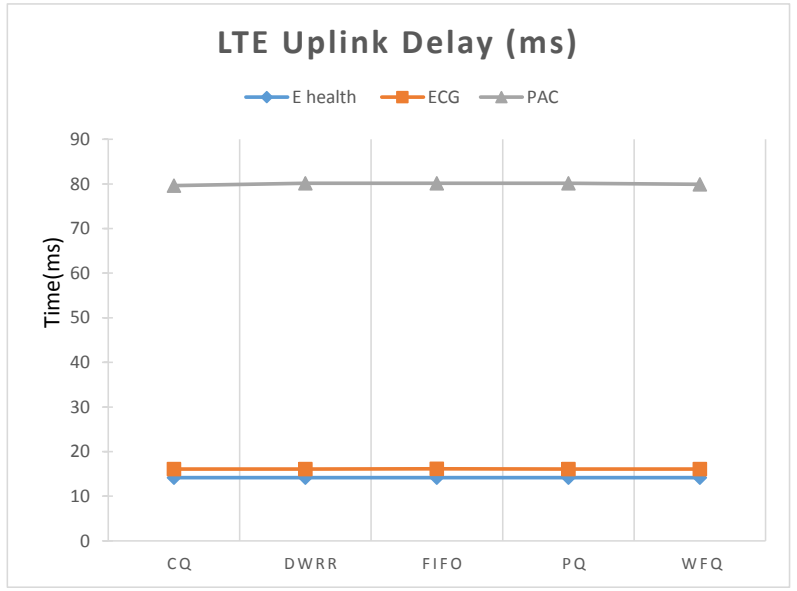

Fig.7.LTE Uplink delay.

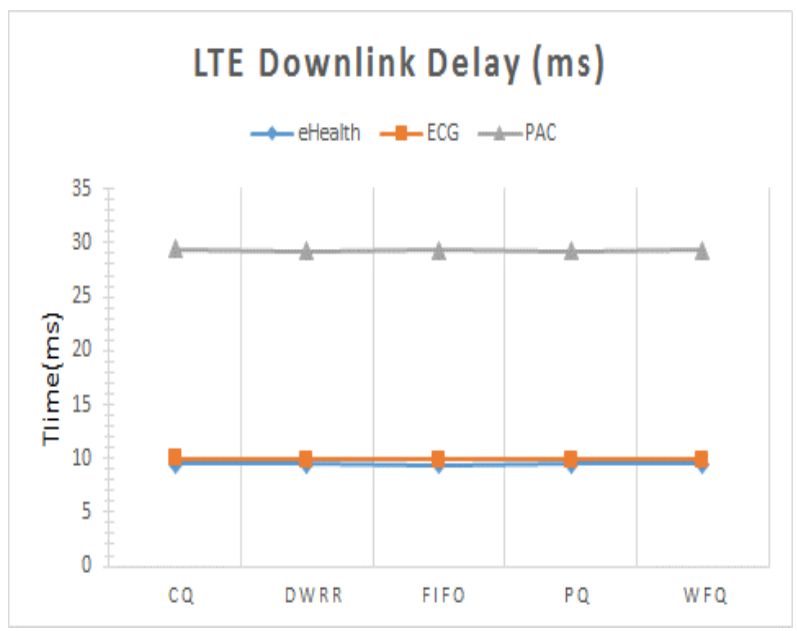

Fig.8.LTE Downlink delay.

\section{CONCLUSION}

In this paper, we investigated three e-healthcare traffic models for medical applications. Moreover, we evaluate the performance of these models and their impact on LTE regular traffic. The FTP users suffer the large delay in file upload time due to low priority as compared e-healthcare devices.

The performance of the voice users under different queuing algorithms does not vary significantly, since they are configured as GBR bearers at the highest MAC QoS class and served at the highest priority. The maximum delay as (CQ), is observed between the algorithms for medical applications.

For future work, we plan to extend the simulation for the new queuing algorithms which would comprise all the advantage of each queuing algorithms. We also intend to evaluate the LTE system performance for other kinds of M2M traffic, such as logistics, surveillance and security, intelligent transportation, city automation, and smart monitoring etc.

\section{REFERENCES}

[1] A.I.A. Jabbar and F. Y.Abdullah, Long Term Evolution (LTE) Scheduling Algorithms in Wireless Sensor Networks (WSN)(2015).Available online: http://research.ijcaonline.org/volume121/number10/pxc3 904626.pdf.
[2] R. J. Haines, P. Kulkarni, and Z. Fan, M2M Communications for E-Health and Smart Grid: An Industry and Standard Perspective, Available online: http://arxiv.org/pdf/1306.3323.

[3] D. K. Mohapatra, e-Health Applications, International Conference on Distributed Computing \& Internet Technology (ICDCIT-2013).

[4] Chen, K.C. Machine-to-Machine Communications for Healthcare. J. Comput. Sci. Eng. 2012, 6, 119-126.

[5] ETSI. Machine-to-Machine Communications (M2M): Functional Architecture. Available online: http://www.etsi.org.

[6] ETSI. Machine-to-Machine Communications: Use Cases of M2M Applications for eHealth. Available online:

http://www.etsi.org/deliver/etsi_tr/102700_102799/1027 $32 /$.

[7] ETSI, TR 102 655, ERM System Reference Document; Short Range Devices (SRD); Low Power Active Medical Implants operating in a $20 \mathrm{MHz}$ band within $2360 \mathrm{MHz}$ to $3400 \mathrm{MHz}, 2008$.

[8] ITU-T Technology Watch Report on E-Health Standards and Interoperability, April 2012, http://www.itu.int/en/ITU-T/techwatch/Pages/ehealthstandards.aspx.

[9] CEN/CENELEC/ETSI, Final Report of the CEN/CENELEC/ETSI Joint Working Group on Standards for Smart Grids, 2010.

[10] ETSI TC M2M, TR 102 764, E-health Architecture: Analysis of User Service Models, Technologies \& Applications supporting eHealth, 2009.

[11] LAN/MAN Standards Committee of the IEEE Computer Society. IEEE Standard for Local and Metropolitan Area Networks-Part 15.6: Wireless Body Area Networks; IEEE STD 802.15.6-2012: Piscataway, NJ, USA, 2012.

[12] M. K. Soraya and M. Singh, Comparison of Various Scheduling Schemes for Voice over LTE Networks, International Conference on Advances Management and Technology (2013).

[13] Nguyen, Huh, E.-N, T.-D., AI-Saffar, A., A dynamic IDbased authentication scheme. In: Sixth International Conference on Networked Computing, Suwon, South Korea, August 16-18, (2010).

[14] LTE for Devices: Requirements, Deployment Phases, and Target Scenarios. In: 12th European Wireless Conference 2011 - Sustainable Wireless Technologies, Vienna, Austria, (2011).

[15] Subash, T. and S. Indira Gandhi, 2006. Performance Analysis of Scheduling Disciplines in IP Networks, in Proceedings of IEEE's WOCN Third International Conference on Wireless and Optical Communication Networks, (2006).

[16] Ahmad Karim, .Voice Performance Over different service Classes under Various Scheduling Techniques, Australian Journal of Basic and Applied Sciences, (2011). 
[17] Demers, A., S. Kasha and S. Shankar, Analysis and Simulation of a Fair Queuing Algorithm, Proceedings of the ACM SIGCOMM, (1989).

[18] E. k., A. S. L., A. A., S. T., M. D. R., L. A., and C. V., A Survey on M2M Systems for eHealth: A Wireless Communications Perspective, Sensors 2014,http://www.mdpi.com/1424-14/10/18009/htm.

[19] Picture Archive and Communication System (PACS). http://www.fresno.ucsf.edu/housestaffportal/documents/ Synapse_PACS.

[20] N. Shea, F. W. Zaki, and S. Keck, Packet scheduling in LTE mobile network International Journal of Scientific,
Volume 4, Issue 6, June-2013. http://www.ijser.org/researchpaper/Packet-scheduling-inLTE-mobile-network.pdf 2016

[21] www.opnet.com.

[22] 3GPP R2-111634, "Report of 3GPP TSG RAN WG2 Meeting \#72bis" ETSI MCC, Dublin, Ireland, January 17 - 21, 2011.

[23] S. Sesia, I. Toufik, and M. Baker, "LTE -The UMTS Long Term Evolution -From Theory to Practice". John Wiley \& Sons, 2011. 